\title{
Discerning a Smile - The Intricacies of Analysis of Post-Neck dissection Asymmetr
}

\author{
Rachael Thomas ${ }^{1}$, Joshua Whittaker ${ }^{2}$, and Jonathan Pollock ${ }^{3}$ \\ ${ }^{1}$ Churchill Hospital \\ ${ }^{2}$ Royal Derby Hospital \\ ${ }^{3}$ Nottingham University Hospitals NHS Trust
}

February 16, 2021

\begin{abstract}
Introduction Iatrogenic facial nerve palsy is distressing to the patient and clinician. The deformity is aesthetically displeasing, and can be functionality problematic for oral competence, dental lip trauma and speech. Furthermore such injuries have litigation implications. Marginal mandibular nerve (MMN) palsy causes an obvious asymmetrical smile. MMN is at particular risk during procedures such as rhytidoplasties, mandibular fracture, tumour resection and neck dissections. Cited causes for the high incidence are large anatomical variations, unreliable landmarks, an exposed course and tumour grade or nodal involvement dictating requisite nerve sacrifice. An alternative cause for post-operative asymmetry is damage to the cervical branch of the facial nerve or platysmal dysfunction. This tends to have a transient course and recovers. Distinction between MMN palsy and palsy of the cervical branch of the facial nerve should therefore be made. In 1979 Ellenbogen differentiated between MMN palsy and "Pseudo-paralysis of the mandibular branch of the facial nerve". Despite this, there is paucity in the literature \& confusion amongst clinicians in distinguishing between these palsies, and there is little regarding these post-operative sequelae and neck dissections. Method This article reflects on the surgical anatomy of the MMN and cervical nerve in relation to danger zones during lymphadenectomy. The authors review the anatomy of the smile. Finally, we utilise case studies to evaluate the differences between MMN palsy and its pseudo-palsy to allow clinical differentiation. Conclusion Here we present a simple method for clinical differentiation between these two prognostically different injuries, allowing appropriate reassurance, therapy \& management.
\end{abstract}

\section{Discerning a Smile - The Intricacies of Analysis of Post-Neck dissection Asymmetry} Keywords:

Marginal mandibular nerve palsy, cervical branch of the facial nerve palsy, Pseudo-paralysis of the mandibular branch of the facial nerve, pseudo-palsy of the mandibular branch of the facial nerve, marginal mandibular nerve, cervical branch of the facial nerve, MMN palsy, lymphadenectomy, smile

\section{Key points:}

- MMN palsy and it's pseudo-palsy have similar presentations

- These conditions have markedly different prognoses for the patient and clinician.

- Both of the MMN and platysmal function are inherently at risk during neck dissection

- The differentiation is a challenge to clinicians, this is evidenced in the literature.

- The authors present a simple, robust method of differentiation between the two palsies, along with the anatomical relevance. Allowing appropriate reassurance, therapy and management can be undertaken

\section{Introduction}


Iatrogenic facial nerve palsy is a concern to the patient and clinician. Not only is the

deformity aesthetically distressing but can also be functionally problematic in terms of oral competence, lip trauma and speech ${ }^{1}$; such injuries are a source of litigation.

Marginal mandibular nerve (MMN) palsy, results in an asymmetrical smile. The MMN is at particular risk during procedures such as rhytidoplasties, mandibular fractures, neck dissections and salivary gland surgery ${ }^{1,2}$. Cited causes for high incidence of MMN palsies are large anatomical variations, exposed course, and tumour grade and node involvement dictating nerve sacrifice.

An alternative cause for post-operative asymmetry is platysma dysfunction caused by damage to the cervical branch of the facial nerve, or direct division of platysma. This usually follows a transient course and tends to recover well. Nerve injury can be of multiple aetiologies: transaction, thermal injury, or retraction, therefore the severity can range from neuropraxia to neurotemesis, complicating the picture. The distinction between MMN palsy and palsy of the cervical branch otherwise termed 'Pseudo-paralysis of the mandibular branch of the facial nerve'2 should therefore be made.

Despite the academic recognition of pseudo-palsies of the MMN, this differentiation is often not emphasised in clinical practice. Establishing the true cause of post-operative smile asymmetry is key to advise the patient on recovery and protect against litigation. This review illustrates the anatomical and clinical considerations when assessing post-operative smile asymmetry.

\section{Anatomy of the MMN and cervical branch of the facial nerve during neck dissections}

Local and regional spread of head and neck tumours occur mostly through the lymphatic system to neck nodes. Neck dissection is not only important for prognosis, but there is evidence to suggest that high lymph node yields equate to better survival even if nodes are negative ${ }^{3}$. National guidelines recommend therapeutic or elective neck dissection based on evidence of nodal metastases, or where occult metastasis risk is over $20 \%$. In head and neck cancer, this means that neck dissection should be considered in all but low stage glottic and oral mucosal malignancy. The practice of cervical lymphadenectomy has changed such that it is now common to perform modified radical necks with selective dissections. The levels dissected depend on location of primary tumour and nodal status.

The marginal mandibular nerve is at risk during dissection of level $\mathrm{Ib}_{\text {and }} \mathrm{IIa}^{4}$ (figure 1). Manoeuvres to protect the nerve have been described such as Hayes-Martin, however, their oncological safety has been contested. This nerve emerges from the caudal border of the parotid gland on its anterior aspect. It exits the gland anterior or inferior to the mandible angle. At this position it lies deep to the parotid-masseteric fascia and the investing layer of the deep cervical fascia ${ }^{5}$. The MMN then courses forward, usually remaining above the inferior border of the mandible, superficial to the anterior facial vessels, deep to the masseteric fascia. If the MMN courses below the level of the mandible it runs over the posterior belly of digastric and the submandibular gland. In this case the nerve is deep to the cervical fascia and takes a curved course ${ }^{6}$. The nerve may run as inferiorly as $3 \mathrm{~cm}$ below the inferior border ${ }^{3}$. Near the mid-body of the mandible, at its inferior border, the nerve perforates the deep cervical fascia to run underneath platysma. It crosses the mandibular border at the anterior border of the masseter ${ }^{6}$, this happens at a point where the nerve intersects with facial vessels. It usually crosses superficial to the facial vein ${ }^{7}$ approximately $3 \mathrm{~cm}$ anterior to the masseteric tuberosity. From here it enters the buccal space to innervate the perioral musculature ${ }^{8}$.

There can be significant variation, in particular it's branching and relationship to lower border of the mandible. There have also been disputes over the depth of the MMN, some authors stating it lies between the deep investing fascia and the platysma ${ }^{7}$. The nerve is thought to be most vulnerable after it penetrates the cervical fascia, coursing cephalad over the mandibular border, close to the facial vessels ${ }^{3}$ and anterior to the masseter (Figure 1).

The cervical nerve is at risk of damage in level II and III (figure 1). This nerve passes inferiorly from anterior aspect of the caudal half of the parotid close to the MMN. By the time the nerves reach the mandible angle their courses have diverged. The MMN runs anterior to the cervical. The cervical nerve usually exits the 
parotid as a single branch and passes posterior to the angle of the mandible, running deep to platysma. Studies have shown the nerve to lie an average distance of $0.83 \mathrm{~cm}$ posterior to the gonion ${ }^{8}$ (figure 1 ).

The nerve usually curves anteriorly; when it reaches the posterior aspect of the submandibular gland it divides into several slender branches, forming a plexus supplying the platysma; this plexus forms anterior to the hyoid ${ }^{8}$. Branches to MMN are rare, but common to the greater auricular nerve ${ }^{8}$. Communicating branches between the transverse cervical nerves occur at the tip of the greater Cornu of the hyoid where an ascending branch of the transverse cervical nerve joins the cervical nerve. The cervical branches tend to be larger than the MMN branches especially after it has received these contributions ${ }^{8}$.

The nerve branching pattern is highly variable. The plexus forms multiple arches spreading across the deep surface of the suprahyoid platysma providing innervation. This variation and division makes the cervical branches inherently vulnerable when raising sub-platysmal flap. The greater branching means injury to a single division is less likely to give functional loss than MMN.

\section{Anatomy of the Smile}

In 1979 differentiation was made between MMN palsy and 'Pseudo-paralysis' of the mandibular branch of the facial nerve, described by Ellenbogen after platysmal face-lift ${ }^{4}$. However, there is often confusion between clinicians, and the literature, distinguishing between the characteristics of individual palsies, and diagnosing a MMN injury ${ }^{9}$. The reason is not only the variable and overlapping innervation, but also the variable function in the peri-oral musculature.

Furthermore, individuals have predominantly different smile types, characterised by Rubin (figure 2). These smile types are associated with different musculature predominance ${ }^{10}$, impacting the effect of palsies in different individuals. Finally, possible arborisation between branches of the MMN and cervical nerve over the mandible have been noted, and so there may be cervical nerve supply to the lower lip depressors. However, this overlap is inconsistent and rare $^{8}$ therefore the significance is disputed.

Rubin classified smiles into three types (Figure 2). In the 'Mona Lisa' smile (a) the zygomaticus major muscle predominates pulling on the corner of the mouth. In the 'canine smile' (b) the dominant muscle is the levator labii superioris. Finally in the full denture smile (c) all muscles are equally activated. Therefore the distinguishing characteristic of the 'full denture smile' is activation of the lip depressors; depressor labii inferioris, depressor anguli oris (MMN) and the platysma (cervical branch). Not only does platysma cofunction with the depressors ${ }^{3}$, but it is also structurally interweaved with them ${ }^{4}$. It makes an important contribution to movement at the mouth corners during opening and smiling. The platysma is supplied by cervical branches of the facial nerve. A patient with this type of smile will have an exaggerated asymmetry with any weakness of depressor function.

The MMN, in addition to innervating the lip depressors, innervates mentalis. The mentalis produces a pout, characterised by eversion of the lower lip $^{9}$. This is perhaps the most helpful distinguishing feature of MMN due to less overlap with other perioral musculature. The obicularis oris may function to aid pursing of the lips. However, the action will be noticeably weaker on the ipsilateral side in MMN injury.

The weakness of lip eversion on the affected side is therefore a clear indicator of a true MMN palsy, whereas asymmetry with retained lip eversion will more likely be related to platysmal dysfunction.

\section{Case examples}

Marginal mandibular nerve palsy

Marginal mandibular nerve palsy, dependent on injury level, will result in both inferior lip depression and lip eversion weakness. Inability to lower the lateral lip downwards and laterally (figure 3a) and eversion of the vermillion border (figure $3 \mathrm{~b}$ ) results in elevation of the ipsilateral side with apparent dropping of the contralateral $^{9}$.

Pseudo palsy of the Marginal Mandibular Nerve 
Transection of the platysma or cervical nerve causing 'pseudo-marginal mandibular nerve palsy' will result weakness of depression, palpable and visible lack of platysmal function below the level of transection (figure 4a). However, pouting will remain intact through mentalis function (figure 4b). Such patients may refrain from a "full-denture type' smile to disguise their palsy.

\section{Conclusion}

Facial nerve palsy is an important consequence of neck dissection. Different palsies have different prognoses, and this is important for patient counselling in terms of long-term prognosis. Despite the differentiation being made academically over 40 years ago, there is a paucity in the literature describing the characteristics particularly in association with neck dissections, where both MMN and platysmal function are inherently at risk.

Here we present a simple method of distinguishing the two palsies, along with the anatomical relevance, so that appropriate reassurance, therapy and management can be undertaken.

Acknowledgment: Thank you to Sciepro/Science Photo Library for underlay picture on figure one.

Data availability statement : Data sharing not applicable to this article as no datasets were generated or analysed during the current study.

\section{References}

1. Amin N, Dixon H, Gibbins N, Lew-Gor S. Marginal Mandibular Nerve Palsy Incidence in Neck Dissection. Otolaryngology Head and Neck Surgery. 2012; 147(2 Suppl):61-61.

2. Ellenbogen R. Pseudo-paralysis of the mandibular branch of the facial nerve after platysmal face-lift operation. Plast. Reconstr. Surg. 1979;63:364.

3. Cheraghlou S, Otremba M, Yu P, Agogo G, Hersey D, Judson B. Prognostic Value of Lymph Node Yield and Density in Head and Neck Malignancies. Otolaryngology Head and Neck Surgery. 2018;158(6):10161023 .

4. Estomba C, Suárez J, González-García J, Larruscain- Sarasola E, Arrizabalaga I, Mariezcurrena X. Marginal mandibular nerve injury during neck dissection of level IIa, and the influence of different types of dissection: diathermy versus cold knife. Otolaryngol Pol. 2018;72(4):21-25.

5. Hazani R, Chowdhry S, Mowlavi A, Wilhelmi B. Bony Anatomic Landmarks to Avoid Injury to the Marginal Mandibular Nerve. Aesthetic Surgery Journal. 2011;31(3) 286-289.

6. Lesley J, Agartala C. Safely Navigating around the Facial Nerve in Three Dimensions. Clin Plast Surg. 2008;35:496-477.

7. Ziarah H, Atkinson M. The Surgical Anatomy of the Marginal Mandibular Distribution of the Facial Nervce. British Journal of Oral Surgeons. 1981;19(3):159-170.

8. Ziarah H, Atkinson M. The surgical Anatomy of the Cervical distribution of the Facial Nerve. British Journal of Oral Surgery. 1981;19: 171-179.

9. Moffat D, Ramsden R. Miehlke A. The deformity produced by a palsy of the marginal mandibular branch of the facial nerve. "The language of facial expression is of extraordinary importance for every human being". J Laryngol Otol. 1977;91(5):401-6.

10. Rubin L. The anatomy of a smile: Its importance in the treatment of facial paralysis. Plast. Reconstr. Surg. 1974;53:384. 


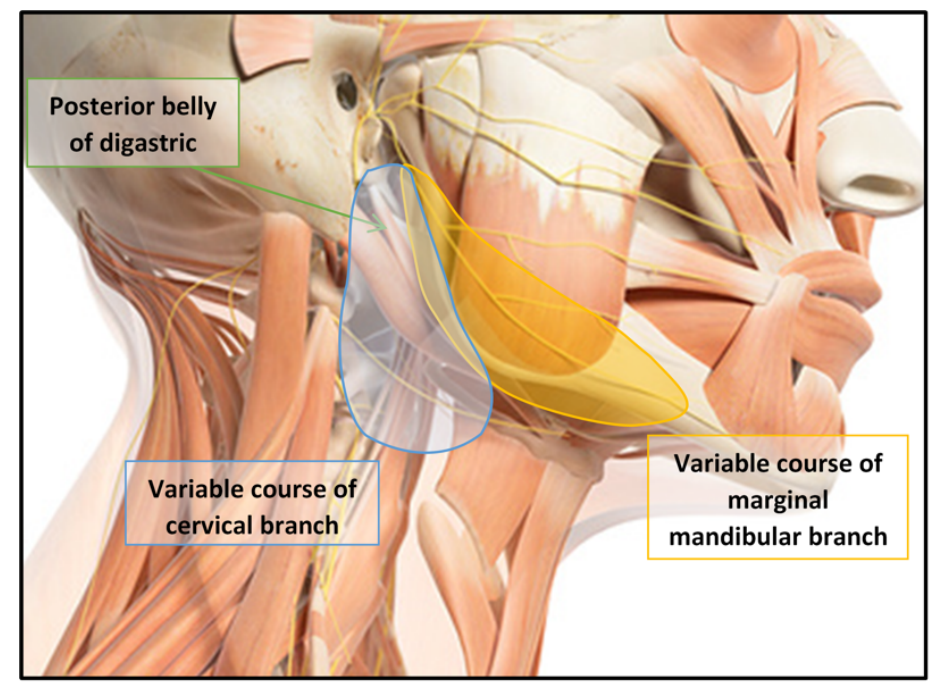

Figure 1. Anatomy of the marginal mandibular nerve and cervical branch of the facial nerve. Credit: Sciepro Photo Library

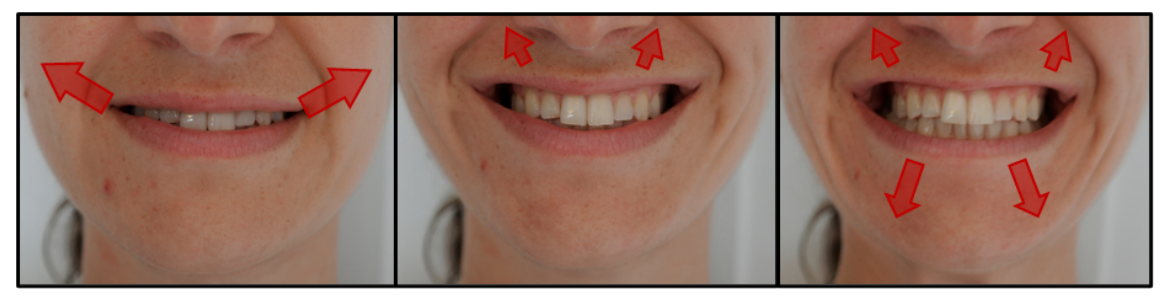

Figure 2. Rubin's smile classifications. a. Mona Lisa, b. The Caine smile, c. The full denture smile (or the complex smile)

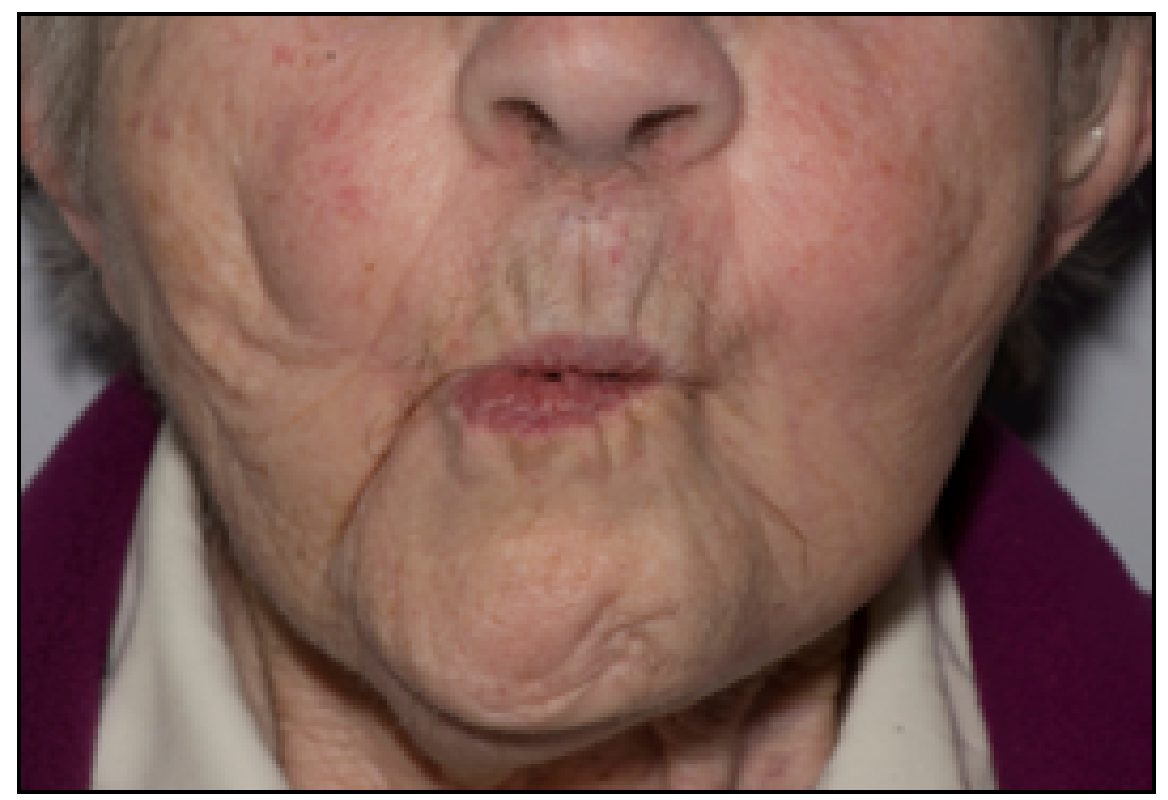



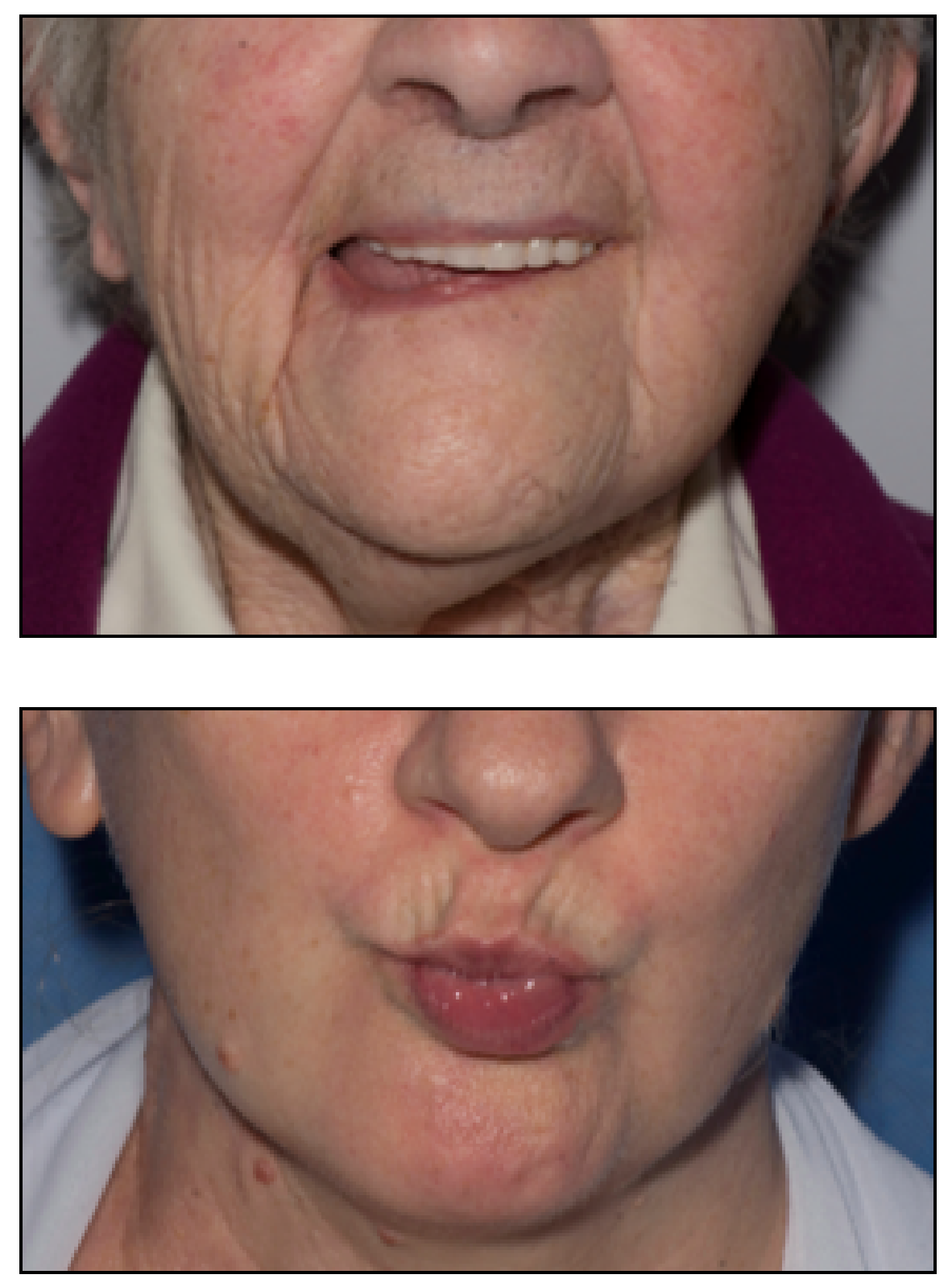


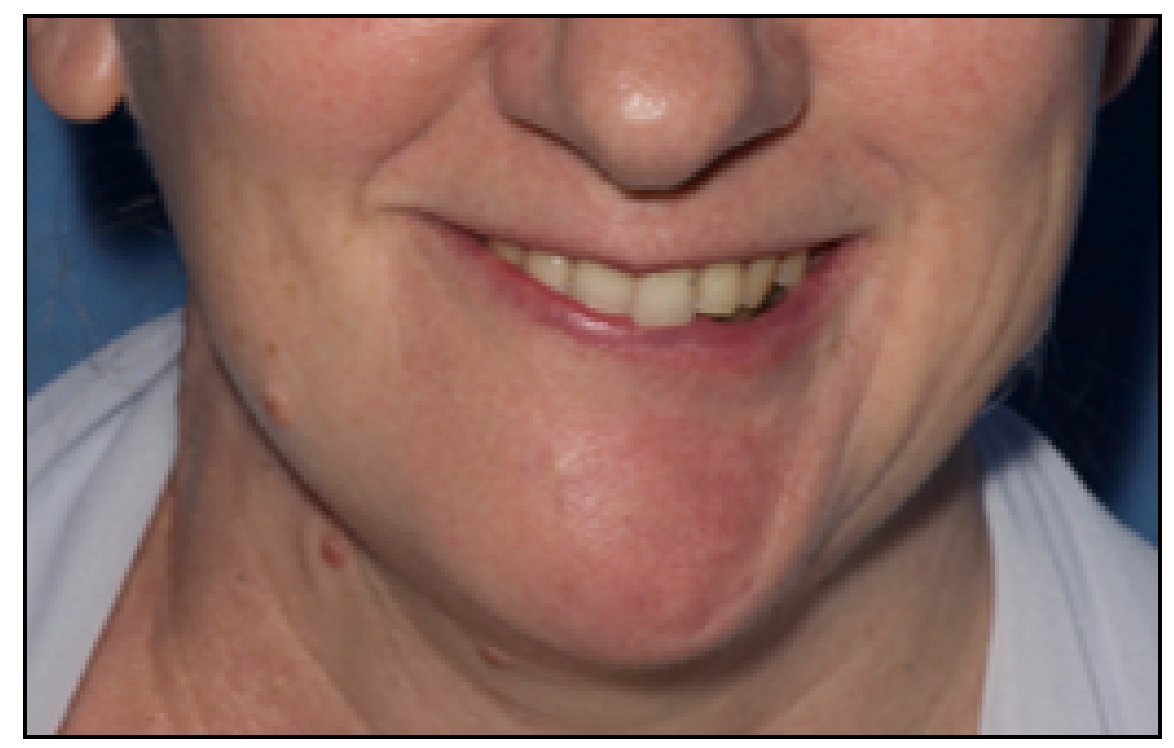

Figure 3. Asymmetrical smile in a patient following left parotid to my and MRND with sacrifice of the marginal mandibular nerve (true palsy). 3a. Paralysis of the lip depressor with apparent drooping of the contralateral side. 3b. Asymmetrical pouting caused by inability to evert the left side of the lower lip

Figure 4. Asymmetrical smile of a patient following a right MRND with preservation of the marginal mandibular nerve (i.e. pseudo-palsy). 4a. Note weakness of the lip depressors on the right side. 4b. The function of the marginal mandibular nerve allows bilateral eversion of the lower lip when pouting. (i.e. pseudo-palsy)

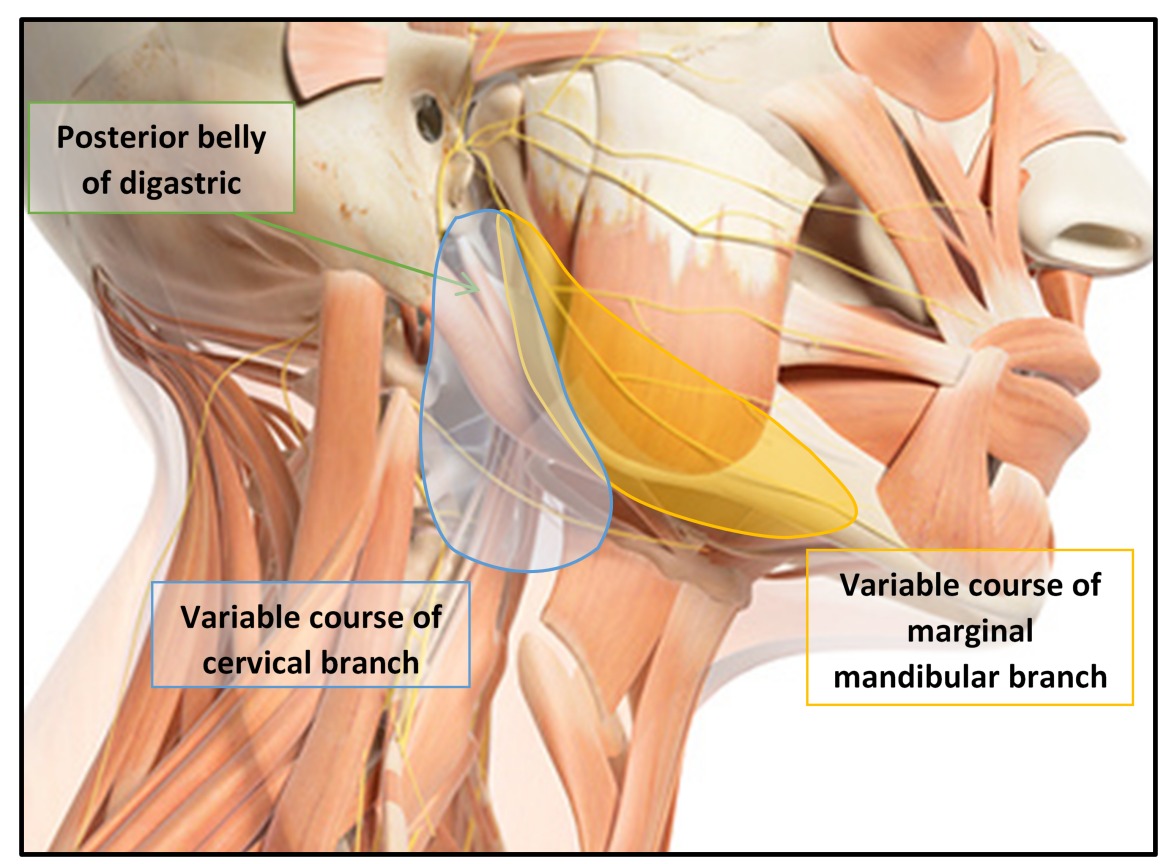



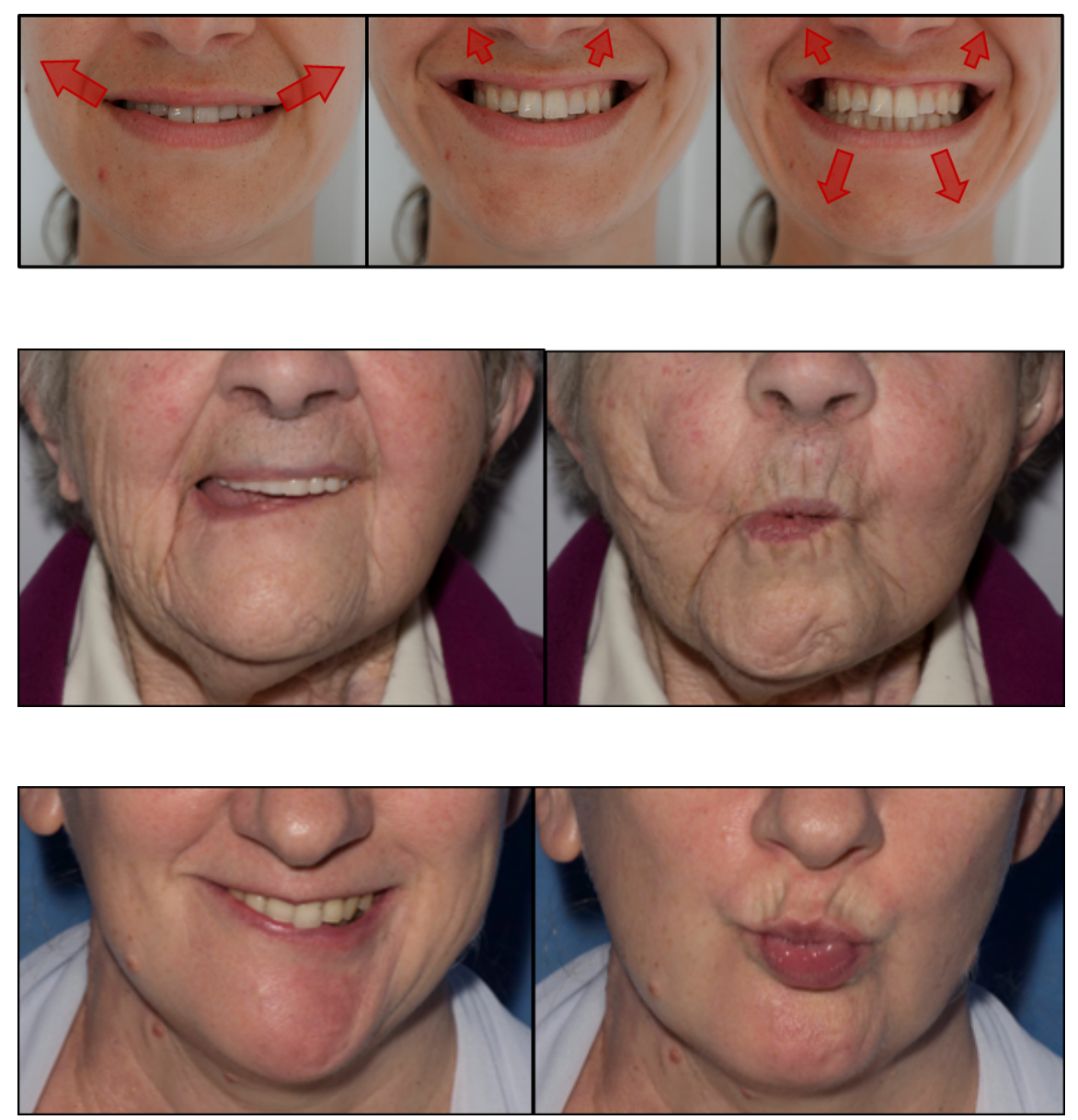